\title{
Navigate: a study protocol for a randomised controlled trial of an online treatment decision aid for men with low- risk prostate cancer and their partners
}

Penelope Schofield ${ }^{1,2,3,4^{*}}$ D, Karla Gough ${ }^{2,5}$, Amelia Hyatt ${ }^{2}$, Alan White ${ }^{2}$, Mark Frydenberg ${ }^{6,7}$, Suzanne Chambers $8,9,10,11$, Louisa G. Gordon ${ }^{12,13,14}$, Robert Gardiner ${ }^{15,16}$, Declan G. Murphy 3,17, Lawrence Cavedon $^{18}$, Natalie Richards ${ }^{2}$, Barbara Murphy ${ }^{19,20}$, Stephen Quinn ${ }^{21}$ and Ilona Juraskova ${ }^{22}$

\begin{abstract}
Background: Active surveillance (AS) is the disease management option of choice for low-risk prostate cancer. Despite this, men with low-risk prostate cancer (LRPC) find management decisions distressing and confusing. We developed Navigate, an online decision aid to help men and their partners make management decisions consistent with their values. The aims are to evaluate the impact of Navigate on uptake of AS; decision-making preparedness; decisional conflict, regret and satisfaction; quality of illness communication; and prostate cancer-specific quality of life and anxiety. In addition, the healthcare cost impact, cost-effectiveness and patterns of use of Navigate will be assessed. This paper describes the study protocol.

Methods: Three hundred four men and their partners are randomly assigned one-to-one to Navigate or to the control arm. Randomisation is electronically generated and stratified by site. Navigate is an online decision aid that presents up-to-date, unbiased information on LRPC tailored to Australian men and their partners including each management option and potential side-effects, and an interactive values clarification exercise. Participants in the control arm will be directed to the website of Australia's peak national body for prostate cancer. Eligible patients will be men within 3 months of being diagnosed with LRPC, aged 18 years or older, and who are yet to make a treatment decision, who are deemed eligible for AS by their treating clinician and who have Internet access and sufficient English to participate. The primary outcome is self-reported uptake of AS as the first-line management option. Secondary outcomes include self-reported preparedness for decision-making; decisional conflict, regret and satisfaction; quality of illness communication; and prostate cancer-specific quality of life. Uptake of AS 1 month after consent will be determined through patient self-report. Men and their partners will complete study outcome measures before randomisation and 1, 3 and 6 months after study consent.

(Continued on next page)
\end{abstract}

\footnotetext{
* Correspondence: pschofield@swin.edu.au; navigate@petermac.org

'Department of Psychology, Swinburne University of Technology, Melbourne,

Victoria, Australia

${ }^{2}$ Behavioural Science Unit, Peter MacCallum Cancer Centre, Melbourne,

Victoria, Australia

Full list of author information is available at the end of the article
}

(c) The Author(s). 2021, corrected publication 2021. Open Access This article is licensed under a Creative Commons Attribution 4.0 International License, which permits use, sharing, adaptation, distribution and reproduction in any medium or format, as long as you give appropriate credit to the original author(s) and the source, provide a link to the Creative Commons licence, and indicate if changes were made. The images or other third party material in this article are included in the article's Creative Commons licence, unless indicated otherwise in a credit line to the material. If material is not included in the article's Creative Commons licence and your intended use is not permitted by statutory regulation or exceeds the permitted use, you will need to obtain permission directly from the copyright holder. To view a copy of this licence, visit http://creativecommons.org/ licenses/by/4.0/. The Creative Commons Public Domain Dedication waiver (http://creativecommons.org/publicdomain/zero/1. 0/) applies to the data made available in this article, unless otherwise stated in a credit line to the data. 
(Continued from previous page)

Discussion: The Navigate online decision aid has the potential to increase the choice of AS in LRPC, avoiding or delaying unnecessary radical treatments and associated side effects. In addition, Navigate is likely to reduce patients' and partners' confusion and distress in management decision-making and increase their quality of life.

Trial registration: Australian and New Zealand Clinical Trial Registry ACTRN12616001665426. Registered on 2 December 2016. All items from the WHO Trial Registration Data set can be found in this manuscript.

Keywords: Low-risk prostate cancer, Decision aid, Management decision, Treatment decision, Active surveillance, RCT, Distress, Quality of life

\section{Background}

Prostate cancer is one of the most commonly diagnosed cancers on a global scale, with incidence rates of 1.1 million during 2012 alone [1]. The vast majority of cases are detected within high-income regions such as Australia, New Zealand, Northern America and Western and Northern Europe. The high incidence rates have been attributed to the widespread use of prostate-specific antigen (PSA) testing [2]. Fortunately, the majority of prostate cancers are localised at the time of diagnosis [3]. Although early detection strategies can reduce the risk of disease progression, they can also result in overtreatment, particularly in men diagnosed with low-risk prostate cancer (LRPC).

Men diagnosed with LRPC may be offered curative treatment options, including radical prostatectomy $(\mathrm{RP})$, radiotherapy (RT) and brachytherapy (BT), or may be managed with routine monitoring called active surveillance (AS). It is estimated that up to $50 \%$ of all prostate cancer cases do not require curative treatment up to 12 years post-diagnosis [4]. Indeed, evidence suggests that there is no survival benefit in providing curative treatments for LRPC at least up to 10 years after diagnosis $[5,6]$. Moreover, these curative treatment options are commonly associated with both shortand long-term side effects such as erectile dysfunction, urinary and bowel incontinence and reduced quality of life [6-8]. This underscores the importance of patient involvement in decision-making regarding the commencement of curative treatment during early-stage disease [9].

AS is now recommended as a preferred management strategy for LRPC [9-13], although rates of AS uptake vary widely around the world [14]. AS is a proactive approach which aims to delay or avoid radical treatments through close monitoring of both the patient and the tumour [15]. AS is now widely accepted as an effective management strategy for LRPC, and clinicians are encouraged to offer AS to eligible patients $[9,13$, $16,17]$. AS has also been shown to be the least costly option for LRPC and increased uptake of AS would lead to substantial cost-savings to the health system [18].

\section{Comparisons in outcomes between management options for LRPC}

A recent examination of cancer mortality statistics revealed a 15 -year survival rate of $95 \%$ for men with LRPC on AS [19]. In terms of age-specific outcomes for AS, 15 -year cancer-specific mortality has been shown to be $5.7 \%$ for men aged $65-74$ years and $10 \%$ for those over 75 [20]. Indeed, 10-year prostate cancer-specific mortality rates have been shown to be similar regardless of whether men undergo AS, RT or RP [6]. However, recent data has raised concerns about compliance with AS protocols, reporting that $75 \%$ of patients do not meet minimum requirements for PSA testing and biopsies to ensure that their cancer is adequately monitored [21].

While survival rates remain similar across management options, impacts on urinary, bowel and sexual function vary significantly. Two recent studies demonstrated significantly greater urinary incontinence and poorer sexual function in men treated with RP compared with those managed by AS [7, 22]. Importantly, men receiving AS report similar outcomes in relation to sexual, urinary and bowel function as men who are not diagnosed with LRPC [7].

\section{Treatment decision making in LRPC}

Management decision making is difficult for men with LRPC. Decision-related distress and confusion are high [23-25], due largely to the variety of management options with no clear best choice and the fact that management decisions are highly dependent on men's individual preferences and lifestyle [26]. For those contemplating AS, anxiety about not receiving radical (i.e. curative) treatment is common [24]. Most men do not consider all management options nor do they make decisions concordant with their personal preferences and values [27, 28]. Partners of men with LRPC also experience high decisional distress and confusion $[15,29]$, and some pressure their partner towards curative treatment [24, 28]. The study anticipates that both men and their partners would benefit from decision-making support, particularly regarding their understanding of the benefits and risks associated with each management option, the risk of the LRPC spreading outside the prostate and the concordance of each option with their personal values and preferences [27].

\section{Decision aids for LRPC}

Decision aids are textual, audio, visual and web-based tools that provide evidence-based information to assist 
patients to consider management options within the context of their own preferences and values. Decision aids guide patients through a deliberative process of actively weighing-up the benefits/costs of available treatment options, thus enabling decision-making that is both evidence-based and considerate of patient preferences and life circumstances [30]. A 2017 Cochrane systematic review demonstrated that decision aids improve knowledge of available treatment options and outcomes, and accuracy of risk perceptions, decrease decisional conflict, increase decisional satisfaction, increase congruency between treatment choice and patient values and improve patient-clinician communication [31]. Level I evidence shows that decision aids reduce the proportion of those who choose surgery in favour of less invasive management options [13].

Several decision aids for LRPC exist, and some of these have shown promise in assisting in decision-making and reducing decisional regret [32-35]. However, two systematic reviews have concluded that none comprehensively addresses the needs of men with LRPC $[26,27]$. The major deficits include incomplete or biassed content, lack of consideration of patient values, inadequate partner involvement, lack of clarification between AS and watchful waiting (WW), lack of theoretical framework, too few examples of patient experiences and inadequate systematic evaluation [26, 27]. Both reviews highlighted an urgent need for high-quality decision aids, which accurately portray AS and are developed in line with International Patient Decision Aid Standards (IPDAS) [26, 27]. Moreover, none of the currently available decision aids have been tailored to the local health care context or are easily accessible by Australian men.

\section{Navigate-an Australian online treatment decision aid for LRPC}

Besides doctors, the Internet is the primary source of information for men with prostate cancer [36]. In 2017, $86 \%$ of Australian households had Internet access at home [37] while $88 \%$ of Australian adults aged 18-75 owned or had access to a smartphone [37]. Provision of an online decision aid enables access from home, promotes rapid and widespread dissemination and enables easy updating to ensure currency of content.

We have developed the Navigate online decision aid which presents up-to-date, unbiased information tailored to Australian men with LRPC in written, graphical and video formats. The Navigate decision aid was informed by a qualitative study which explored the experiences of 21 men with LRPC who had been on AS for at least 3 months, and 14 of their partners [24]. The findings confirmed that partners were highly involved in management decision-making and highlighted the need for consistent and accurate discourse surrounding LRPC diagnosis and management options. Men and their partners emphasised the need for balanced information to facilitate informed and values-based treatment decisions [24]. Navigate was co-designed by consumers and a multidisplinary team, is evidence-based and theoretically underpinned and complies with IPDAS criteria. Table 1 displays the key features of the website and example graphic images.

\section{Methods}

\section{Study aims and hypotheses}

The aims of this study are to evaluate the impact of Navigate for men with low-risk prostate cancer and their partners on uptake of AS as a first-line management option; men's preparedness for decision-making; men's decisional conflict, regret and satisfaction; the quality of men's illness communication; and men's prostate cancer-specific quality of life. The secondary aims are to estimate study arm differences in men's anxiety; explore the impact of Navigate on partner's decisional conflict, regret and satisfaction, and quality of illness communication; assess the healthcare cost impact and costeffectiveness of Navigate (economic sub-study); and determine the specific patterns of use of Navigate (web analytic sub-study).

Specific hypotheses are that a higher proportion of men randomised to Navigate will select AS as a first-line management option when compared with men randomised to the control group, and men randomised to Navigate will feel better prepared for decision-making, experience lower levels of decisional conflict and regret and higher levels of decisional satisfaction and report better quality of illness communication and better prostate cancer-specific quality of life when compared to men randomised to the control group.

Research questions, rather than hypotheses, were developed for secondary aims, since no specific predictions were made. Research questions relevant to the main study include:

Do men randomised to Navigate report higher or lower levels of anxiety than men in the control group?

Do the partners of men randomised to Navigate experience lower levels of decisional conflict and regret, and higher levels of decisional satisfaction when compared with the partners of men randomised to the control group?

The research question for the economic sub-study is:

What is the cost-effectiveness of the decision-aid intervention compared with usual care for men newly diagnosed with prostate cancer?

The research questions for the web analytic study include:

What are the general patterns of use of the website; are there any identifiable areas for improvement from a user experience perspective? 
Table 1 Key features of the Navigate online decision aid
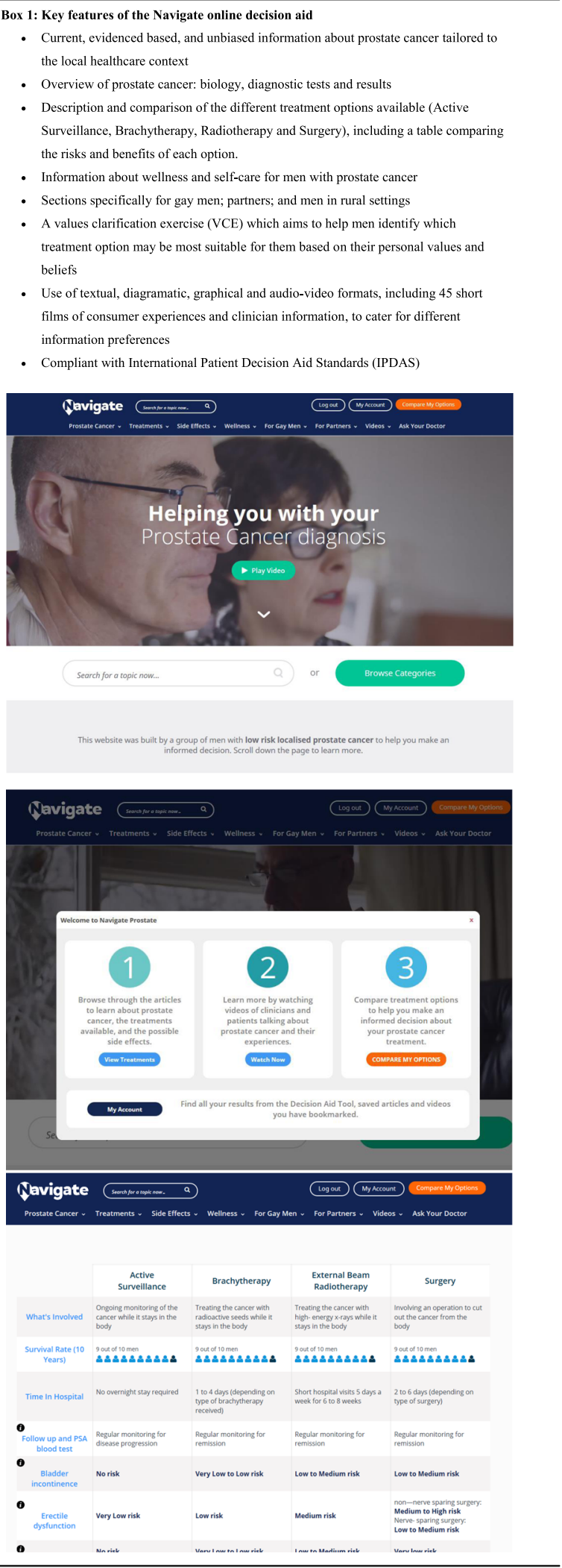

Do men diagnosed with prostate cancer use the website differently compared with partners of these men?

What are the patterns of use across individuals relating to the values clarification exercise?

Do patterns of use relate to the primary and secondary outcome variables?

\section{Trial design and randomisation}

This study will use a parallel group, prospective randomised controlled trial (RCT) with one baseline and three post-baseline assessments (i.e. follow-ups 1, 2 and 3), along with economic and web analytics substudies. In this study, 'partner' refers to the support person nominated by the patient. Both patients and their partners (if one is nominated) will be invited to participate. Following informed consent and completion of baseline study measures, participating patients and their partners will be randomly assigned to Navigate or the usual care arm with a one-to-one allocation, stratified by recruitment site. Randomisation will be undertaken remotely and independently by the trial coordinator using a purposebuilt Microsoft Access randomisation database. After a participant (patient/partner) has been enrolled and completed baseline measures, the trial coordinator uses the randomisation database to assign the participant to the intervention or usual care arm and informs them via email of the experimental allocation. Post-baseline assessments will occur approximately 1, 3 and 6 months after consent. Participants will be informed of their allocation after completing baseline questionnaires. Researchers involved in data collection will be blinded to group allocation. Statisticians will be unblinded to allocation before preparation of the participant flow diagram and outcome analysis. Ethical approval was received from the Peter MacCallum Cancer Centre Human Research Ethics Committee (HREC16 PMCC114). Figure 1 displays the enrolment, group allocation, intervention and assessment schedule.

\section{Participants and study setting}

Australian patients will be eligible to participate regardless of whether or not their partners participate, whereas partners are not eligible without a participating patient.

\section{Eligibility criteria}

Men will be eligible to participate in this trial if they are 18 years or older; have been diagnosed with LRPC in the last 3 months and have yet to make a treatment decision; have been deemed eligible for AS by their treating clinician; and have Internet access and sufficient English to complete study requirements and use the Navigate website. Men will be ineligible if they have a severe psychiatric or cognitive disorder or are too unwell to 
participate as deemed by their treating clinician, selfreport, or the research team at the time of approach.

Partners are eligible to participate in this trial if they are 18 years or older, are the designated partner identified by the consenting patient and have Internet access and sufficient English to complete study requirements and use the Navigate website.

Participating sites include five Australian treatment centres within Victoria (Peter MacCallum Cancer Centre, Western Hospital, Cabrini Hospital, Alfred Hospital, Austin Hospital) and two in Queensland (Royal Brisbane and Women's Hospital, Redcliffe Hospital). Men with LRPC and their partners from either public or private health care settings are also able to self-refer online to the trial.

\section{Intervention}

Intervention participants are emailed login details to access the Navigate online decision aid which is hosted on the Prostate Cancer Foundation of Australia website. Using Navigate, men and their partners are led through information on each treatment option for LRPC with its potential benefits and side-effects. An interactive values clarification exercise assists the men with weighing up the advantages and disadvantages of each management option to clarify what matters to them and to guide their preferences.

\section{Usual care}

Usual care participants are provided with minimal information to fulfil ethical obligations and emailed a link to the Prostate Cancer Foundation of Australia website page for LRPC treatments. This website provides brief information on LRPC and treatment options, but differs from the Navigate website in that it does not include comparisons of the pros and cons of each management option, a values clarification exercise, nor the presentation of material in graphical or multimedia formats.

\section{Recruitment and consent}

Potentially eligible men will (i) be identified by their treating clinician/nurse or the site investigator using screening clinic lists or (ii) self-refer to the website via digital marketing strategies. The treating clinician can also refer interested patients directly to the study team. Once men and their partners provide consent and complete baseline questionnaires, they are provided with the appropriate website link as per their group allocation. Non-consenters are asked for de-identified demographic and clinical details. Attrition will be monitored and reasons for withdrawal recorded.

\section{Hospital recruitment and consent}

Eligible men are approached after their diagnosis by research nurse/assistant, consistent with the agreement of those patients' treating doctors. The research nurse/assistant reviews the Patient Information and Consent Form (PICF) with interested patients and patients provide online consent via the Qualtrics survey platform. Using the same consent procedure, partners are invited to participate. Consenting patients are also asked to provide written consent for the study team to obtain data from the Medicare Benefits Scheme and Pharmaceutical Benefits Scheme databases.

For patients or partners who are unsure about participating or do not have time to complete the PICF in clinic, a research team member is able to contact the patient/partner by telephone within a week to determine participation status. If the patient/partner agrees to participate, they are emailed a personalised PICF link.

\section{Self-referral, recruitment and consent}

The study is being promoted using digital marketing and community engagement strategies (Google Ads, Facebook, social media, blogs, commentary articles). Men who self-refer to the study are asked to complete an online Expression of Interest form including the details of their treating clinician who will be contacted by the study team to confirm eligibility. If eligible, a research nurse/assistant will undertake the consent process with the patient over the telephone.

\section{Clinician referral, recruitment and consent}

The treating clinician can also refer their patients directly the study confirming they meet the eligibility criteria and they have discussed the trial with his/her patient who is happy to be contacted. A research nurse/ assistant is then available to undertake the consent process with the patient over the telephone.

\section{Procedure and assessments}

After consent, the research team emails a link to access this questionnaire to be completed at home. The online questionnaires were created by the research team within Qualtrics, an online program which prevents skipping questions. A reminder telephone call is made if the study measures have not been completed within $48 \mathrm{~h}$. However, if the participant exits the form without completing the entire questionnaire, they are not followed up to obtain the remaining data.

Follow-ups 1, 2 and 3 are emailed to participants respectively at 1, 3 and 6 months post-consent. If followup questionnaires have not been completed within 2 weeks, a reminder telephone call is made. After completion of follow-up 3, participants are contacted via email and telephone to confirm trial completion and to 


\begin{tabular}{|c|c|c|c|c|c|}
\hline & \multicolumn{5}{|c|}{ Study Period } \\
\hline & \multirow{2}{*}{$\begin{array}{c}\text { Enrolment } \\
\text { Within } 3 \\
\text { months of } \\
\text { LRPC } \\
\text { diagnosis }\end{array}$} & \multirow{2}{*}{$\begin{array}{c}\text { Allocation } \\
0 \\
0\end{array}$} & \multicolumn{3}{|c|}{ Assessment } \\
\hline TIMEPOINT & & & 1 month & 3 months & 6 months \\
\hline \multicolumn{6}{|l|}{ ENROLMENT: } \\
\hline \multirow{4}{*}{$\begin{array}{r}\text { Eligibility screen } \\
\text { Informed consent } \\
\text { Baseline } \\
\text { Questionnaire } \\
\text { Allocation }\end{array}$} & $\mathrm{X}$ & & & & \\
\hline & $\mathrm{X}$ & & & & \\
\hline & $\mathrm{X}$ & & & & \\
\hline & & $\mathrm{X}$ & & & \\
\hline \multirow{3}{*}{$\begin{array}{r}\text { INTERVENTIONS: } \\
\text { Navigate DA } \\
\text { Control with PCFA } \\
\text { website }\end{array}$} & & & & & \\
\hline & & $\mathrm{X}$ & & & \\
\hline & & $\mathrm{X}$ & & & \\
\hline \multicolumn{6}{|l|}{ ASSESSMENTS: } \\
\hline \multirow{2}{*}{$\begin{array}{r}\text { Decision-making } \\
\text { preparedness } \\
\text { Illness } \\
\text { communication }\end{array}$} & $\mathrm{X}$ & & $\mathrm{X}$ & & \\
\hline & $\mathrm{X}$ & & $\mathrm{X}$ & & \\
\hline \multirow[t]{2}{*}{ Decisional Conflict } & & & $\mathrm{X}$ & & \\
\hline & & & $\mathrm{X}$ & & \\
\hline $\begin{array}{l}\text { Decisional } \\
\text { satisfaction }\end{array}$ & & & & $\mathrm{X}$ & $\mathrm{X}$ \\
\hline \multirow[t]{2}{*}{ Decisional regret } & & & & $\mathrm{X}$ & $\mathrm{X}$ \\
\hline & $\mathrm{X}$ & & $\mathrm{X}$ & $\mathrm{X}$ & $\mathrm{X}$ \\
\hline
\end{tabular}

Note: DA=Decision Aid; PCFA=Prostate Cancer Foundation of Australia

Fig. 1 Enrolment, group allocation, intervention and assessment schedule. Note: DA, decision aid; PCFA, Prostate Cancer Foundation of Australia

thank the participant for their time. The trial flow chart is presented in Fig. 2.

\section{Main study measures}

\section{Demographic and clinical information}

Demographic information collected from patients and partners includes age (in years), marital status, postcode, highest education level, occupation and ethnic origin.

Clinical information (Gleason score and PSA) is obtained from the referring site or the referring clinician or collected via a medical record audit.

\section{Primary outcome}

The primary outcome is self-reported uptake of AS as the first-line management option for LRPC assessed as a percentage (AS or curative treatment option) at followup 1.

\section{Secondary outcomes}

Preparedness for decision-making is assessed at follow-up 1 with the preparedness for decision-making scale (PrepDM). The PrepDM total scale measures the perceived usefulness of the decision aid in preparing the patient to communicate with his doctor(s) to make a health decision and has shown acceptable internal consistency (Cronbach's $\alpha=0.92$ to 0.96 ), and Item Response Theory analyses demonstrated that all ten scale items function well [38].

Decisional conflict is assessed at follow-up 1 with the 16-item Decisional Conflict Scale (DCS). The DCS 
Clinician/Nurse/RA identifies eligible patient or patient self-identifies

Inclusion: aged $\geq 18$ years, diagnosed with LPC within the last 3 months, yet to make a treatment decision, deemed eligible for AS by their treating clinician or meet the NCCN guidelines for AS; have access to the Internet, able to read and write English.

Exclusion: has a severe psychiatric or cognitive disorder, or too unwell to participate as deemed by their treating clinician/nurse, study RA or by self-report.

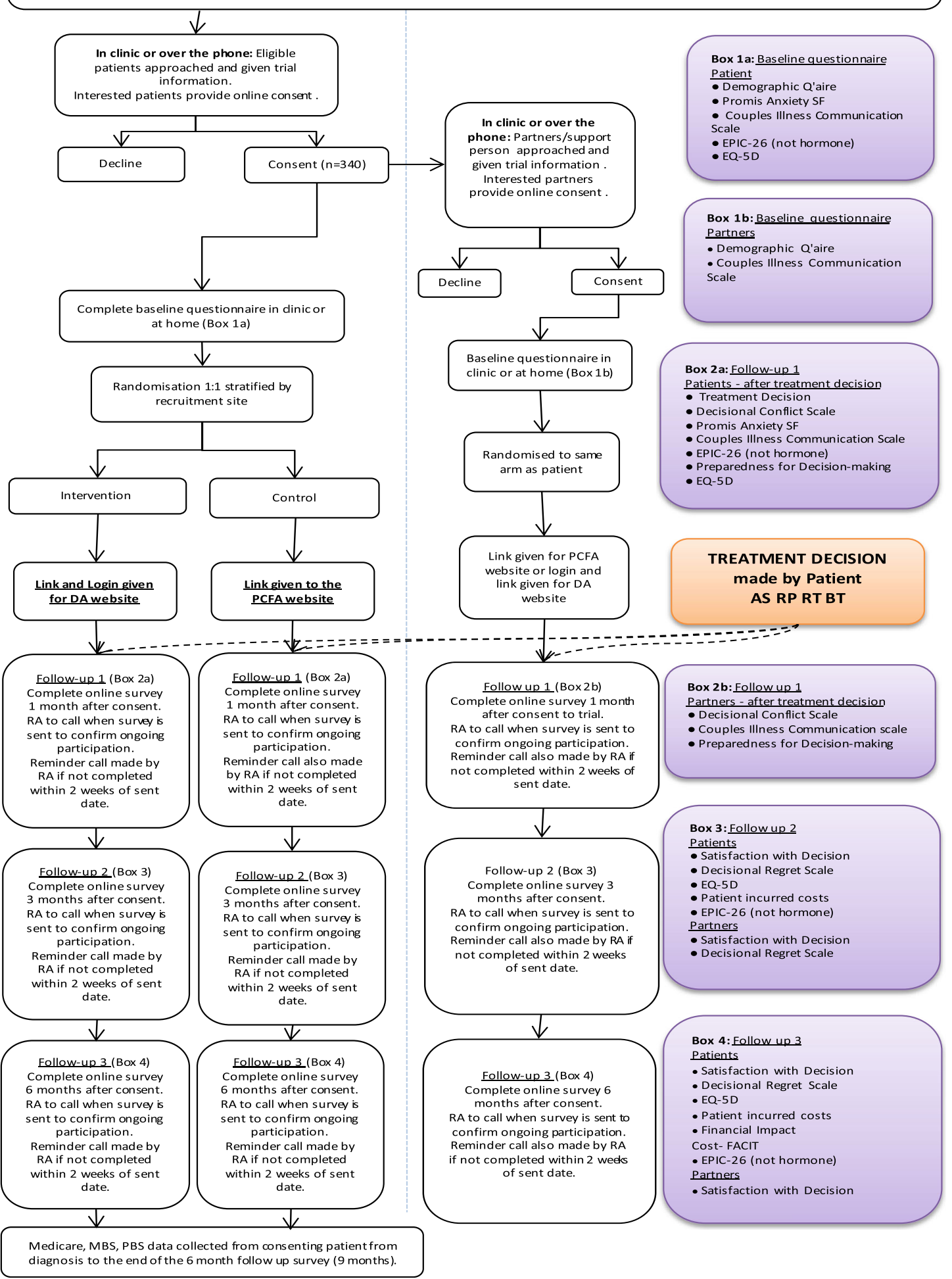

Fig. 2 Study trial flow chart 
measures patients' perceptions of uncertainty, being uninformed and unsupported and having unclear values in decision making. The DCS is suitable for use with cancer patients and has shown acceptable internal consistency (Cronbach's $\alpha=0.78-0.92)$, test-retest reliability ( $r=$ 0.81 ) and responsiveness (longitudinal validity) [39].

Decisional satisfaction is assessed at follow-up 2 and, for patients only, follow-up 3 with the 6-item Satisfaction with Decision (SWD) measure. The SWD has shown acceptable internal consistency (Cronbach's $\alpha=$ $0.86)$, discriminant validity and responsiveness [31, 40].

Decisional regret is assessed at follow-up 2 and, for patients only, follow-up 3 with the 5-item Decisional Regret Scale (DRS). The DRS has shown acceptable internal consistency (Cronbach's $\alpha=0.81-0.92$ ) and convergent validity with related measures $(r=0.31-0.60)$ and responsiveness [31].

The quality of men's and partners' illness communication is assessed at baseline and follow-up 1 with the 4item Couples' Illness Communication Scale (CICS). The CICS was adapted for use in prostate cancer populations. The original scale has shown acceptable internal consistency (Cronbach's $\alpha=0.80$ ) and convergent validity with other measures of dyadic adjustment [41].

Anxiety is assessed at baseline and follow-up 1 with the 7-item PROMIS Emotional distress-Anxiety 7a short-form. The Anxiety 7a is a standardised, valid and precise measure specifically developed for use in clinical oncology research [42].

Prostate cancer-specific quality of life is assessed at baseline and every follow-up with the 26-item Expanded Prostate Cancer Index Composite short-form (EPIC-26). The EPIC-26 comprises four subscales: urinary, bowel, sexual and hormonal. The hormonal subscale is not relevant in this context, so will not be administered. Subscales have shown acceptable internal consistency (all Cronbach's $\alpha>0.70$ ), test-retest reliability (all $r>0.69$ ) and responsiveness when used independently [43].

\section{Sample size}

The sample size calculation was based on $80 \%$ power, a two-sided $\alpha=0.05$ test, and a difference in uptake of the AS treatment option of $15 \%$. Assuming $65 \%$ uptake in the usual care group [3] and $80 \%$ uptake in the intervention group (a conservative assumption), the required sample size is 272 patients (136 in each study arm). Our sample size calculation was performed with PASS version 16. Allowing for $10 \%$ attrition, a total sample size of 304 participants is required.

\section{Statistical analysis}

All analyses will be performed using Stata 16. Prior to formal analysis, descriptive statistics and graphical displays will be used to identify missing and out-of-range values, assess distributional assumptions and identify outliers.

Recruitment bias will be assessed by comparing demographic and clinical characteristics of consenters and non-consenters using $t$ tests (or Mann-Whitney) and chi-squared (or Fisher's exact) tests as appropriate. Differential attrition will be assessed by comparing baseline characteristics of drop-outs and continuing participants using the same statistical tests.

\section{Outcome analysis}

The primary analysis will follow the intention-to-treat principle and all study participants will be analysed as part of the study arm to which they were randomised in all outcome analysis. Missing data will be imputed using multiple imputation, redrawing 50 samples. Pearson's chi squared test will be used to analyse study arm differences in the primary outcome. Linear regression will be used to analyse study arm differences for preparedness for decision-making at followup 1, illness communication at follow-up 1 and anxiety at follow-up. Each model will be adjusted for baseline and group assignment. Decisional conflict (follow-up 1), satisfaction (follow-ups 2 and 3) and regret (follow-ups 2 and 3) will be analysed using linear regression with group assignment as the independent covariate (equivalent to a test). The effect between groups and significance will be assessed using the beta-coefficient and $p$ value corresponding to the group assignment term. Finally, prostate cancer-specific quality of life will be analysed with a random effects mixed model. The independent variables will be group assignment, time (coded 0/1) and the interaction term group assignment BY time. The effect and between groups and significance will be assessed using the beta-coefficient and $p$ value corresponding to the interaction term. All results will be presented with $95 \%$ confidence intervals and a $p$ value less than 0.05 (two-sided) will be deemed to be statistically significant.

\section{Exploratory analyses}

Participants will be analysed as part of the study arm to which they were randomised in all exploratory analysis. Regression (similar to the above) will be used to analyse study arm differences in the following exploratory outcomes: men's anxiety (at follow-up 1), partners' illness communication with men (at follow-up 1), partners' decisional conflict (follow-up 1), partner's satisfaction (follow-ups 2 and 3) and partner's regret (follow-ups 2 and $3)$. The effect between groups and significance will be assessed using the beta-coefficient and $p$ value corresponding to the group assignment term. 


\section{Health-economic sub-study}

The aim of the economic sub-study is to assess the financial burden and cost-effectiveness of the Navigate online decision aid in the Australian context. This will compare the costs and patient outcomes of intervention versus usual care aligning to the goals of the planned RCT. The analysis will take a societal perspective and include Medicare data for all patient participants to assess healthcare costs to government and patient out-ofpocket expenses from participant surveys.

Health utility data will be collected at baseline and follow-ups 2 and 3. The 5-item EuroQol-5D (EQ-5D-5L) will be used, which is a standardised measure of health status developed specifically for economic evaluation and is suitable for cancer patients. It has shown acceptable internal consistency (Cronbach's $\alpha=0.71$ ), testretest reliability $(\mathrm{kappa}=0.7)$, convergent validity $(r>$ $0.49)$, discriminant validity and responsiveness [44, 45].

Patient health care costs will be reported by patients at follow-ups 2 and 3. These include out-of-pocket expenses for all medical services, as well as costs for travel, accommodation and income lost from interrupted employment. The Patient Costs Questionnaire (PCQ) is purpose-built for Australian men with prostate cancer [46] and includes items such as general practice and specialist visits, counselling and support services and sexual and incontinence aids. Follow-up 3 will also include the COST-FACIT questionnaire [47] capturing financial hardship and additional questions used previously by men with prostate cancer relating to early retirement and financial situation.

At the end of the data collection period, Medicare data on all services and medicines for all consenting participants will be accessed. With this data, the types and dollar amounts exchanged for health services, community services and medicines for prostate cancer and other diseases will be assessed, including medical services for privately-insured patients. The main economic outcome will be incremental cost per quality-adjusted life year for the Navigate tool versus usual care. Methods for this economic evaluation will be governed by standardised guidelines in modelling studies and economic evaluations $[48,49]$.

\section{Web analytics sub-study}

Website analytics will be used to indicate preferred modes of interaction, which aspects users (participants) find most engaging and how users prefer to access information on the Navigate website. This provides indicative answers to specific questions, such as how comprehensively users consume the information regarding the different management options before submitting preferences on decisions. Analysis of user behaviour may lead to a better understanding of the effectiveness of the decision tool, as well as potentially identifying areas for improvement. Planned data capture includes click information, pages visited, time spent on each page, information viewed and/or downloaded and values clarification exercise responses. Since data is associated with user ID, behaviours can be linked to demographic characteristics such as age, sexual orientation, or user type (i.e. patient or partner).

Analysis will be restricted to the intervention group of the randomised study since the focus of this sub-study is the characteristics and effectiveness of the web-based decision tool. Effects to be investigated include informational value of pages (determined by number of visits and time spent), as well as complex behavioural effects such as the amount of information consumed before coming to decisions. Variance across demographic subgroups will be measured using appropriate statistical metrics (e.g. ANOVA, chi-squared) to determine demographic-based preferences. Data mining techniques (e.g. association-rule mining) will be applied to attempt to determine other associations between behaviour characteristics (e.g. consuming certain information leading to certain decisions and/or outcomes).

\section{Data storage, management and future use}

All data is kept in password-protected databases. Nonidentifiable participant data is separated from databases linking names with participant identifiable details. Once the study is completed, this database with identifiable details will be destroyed; the remaining data will be retained indefinitely. With the exception of the Medicare data, participants who consent to this study will also be consenting to the use of their data for future unspecified research. To obtain access to the data obtained through this project, investigators will have to have their projects approved by the HREC of their host institution and by Peter Mac Cancer Centre HREC.

\section{Discussion}

With the increase in diagnoses of LRPC $[1,50]$, there is an urgent need for resources to support patients and their partners in their management decision making and, specifically, to reduce the choice of potentially unnecessary radical treatments with inherent significant side effects $[26,27]$. Confusion, anxiety, distress and decisional regret are common in men with LRPC [23-25] and in their partners which may be enduring $[15,24,28,29]$. Indeed, men with LRPC and their partners express the need for unbiased information about potential benefits and risks of their management options, to help them informed decisions which are aligned their own personal preferences and values [24].

The Navigate online decision aid has the potential to increase the choice of AS to manage LRPC, thereby 
avoiding or delaying radical treatments. In addition, Navigate has the potential to reduce patients' and partners' confusion and distress in management decision making, reduce decisional regret, increase decisionmaking preparedness and decisional satisfaction and improve prostate cancer-specific quality of life. Navigate will be made available to all Australian men diagnosed with LRPC to support their management decision making.

Besides the potential ramifications on quality of life for men who receive treatment with curative intent, management of LRPC exerts a substantial financial burden to governments, hospitals and men affected by this condition which is increasing annually [18]. Approximately 25\% of Australian prostate cancer patients diagnosed have LRPC [3, 11]: approximately 5500 per year. If this intervention is successful in achieving the minimal expected difference of $15 \%$, which represents 825 men selecting AS in preference to the most common definitive treatment approach (i.e. radical prostatectomy), this could present an annual cost saving of at least AU\$6.1 million to Australians (difference in average cost per patient of AU\$1400 for government and AU\$2300 for out of pocket) [18]. As prostate cancer diagnoses and health care costs escalate, these projected cost savings will grow. Therefore, this project has the potential to reduce both the financial costs to the government and personal costs to the large and growing population of men with LRPC.

The findings of this study will be disseminated via publications in peer-reviewed journals and by engagement with clinicians, media, government and consumers. In particular, we will promote the outcomes of this study amongst the broader medical and consumer community to improve treatment paradigms and approaches to supportive care for patients with LRPC and their partners. This will include providing the Navigate online decision aid as standard care for patients diagnosed with prostate cancer via the Prostate Cancer Foundation of Australia website, if ultimately proved effective.

\section{Trial status}

The protocol version number is HREC16PMCC114 NAVIGATE_Protocol_V8_27/03/2020. Patient recruitment opened on May 2017, and it is estimated that it will cease in December 2020.

\footnotetext{
Abbreviations

LRPC: Low-risk prostate cancer; AS: Active surveillance; RCT: Randomised controlled trial; RT: Radiotherapy; BT: Brachytherapy; RP: Radical prostatectomy; WW: Watchful waiting; IPDAS: International Patient Decision Aid Standards; MRC: Medical Research Council; PICF: Participant Information and Consent Form; MBS: Medicare Benefits Scheme; PBS: Pharmaceutical Benefits Scheme; DCS: Decisional Conflict Scale; SWD: Satisfaction With Decision measure; DRS: Decision Regret Scale; CICS: Couples IIIness
}

Communication Scale; EPIC-26: Expanded Prostate Cancer Index Composite short-form; EQ-5D: EuroQol-5D

\section{Acknowledgements \\ The authors wish to thank the following people involved in the trial: Lama Karroum, Paula Rodger, Trista Koproski, Judith Hankin, Carla D’Amico, Marianne Heppell, Sarah Johnston, Sarah Mann and Cindy Ogluszko. We thank all the participants of Navigate and consumer advocates who were involved.}

\section{Trial sponsor}

Funding will be managed by study sponsor: Swinburne University of Technology, Melbourne, Australia. The trial sponsor provides infrastructure and enables the running of the trial, but has no influence over study design; collection, management, analysis and interpretation of data; writing of the report; and the decision to submit the report for publication.

\section{Trial governance}

A steering committee comprising of all investigators and project staff oversee all aspects of the trial including access to the final trial dataset and statistical code. A project committee consisting of all project staff, the principal investigator and other investigators (as required) oversees the dayto-day management of the trial including adherence to protocol and ethical standards, training of staff and data management, accuracy and completeness. A data monitoring committee or interim analyses are not required as harm to participants is not anticipated. All personal information will be kept strictly confidential as per study protocol. Details are available in the full protocol which can be obtained by the corresponding author.

\section{Authorship}

Authorship of all publications arising from this study will follow the International Committee of Medical Journal Editors recommendations. Professional writers will not be used.

\section{Authors' contributions}

PS conceptualised the research idea, led the funding award, contributed to the implementation of the trial in Victoria and provides ongoing trial oversight. KG contributed to the study design and statistical analysis. $\mathrm{AH}, \mathrm{AW}$ and NR contributed to the study design, procedures and implementation of the trial. MF, DM and FG provide clinical expertise and assist with promotion of the trial. SC and IJ provide clinical and decision making intervention expertise. LG provides expertise and manages the health economic component of the trial. LC oversees the information technology aspects of the projects. BM contributed to the conceptualisation of this paper. CS contributed to developing the website and to the writing of the manuscript All authors have contributed to writing of this manuscript. The authors read and approved the final manuscript.

\section{Funding}

This trial is supported by the National Health and Medical Research Council (NHMRC). The funder provided the research funds and has no role in the collection, analysis, interpretation, or publication of this study.

\section{Availability of data and materials}

The datasets generated and/or analysed during the current study are available from the corresponding author on reasonable request and in accordance with ethical restrictions imposed by the Ethics Committees that approved this study.

\section{Ethics approval and consent to participate}

The scientific title of this study is Navigate: Randomised Controlled Trial of an online decision aid for men with low-risk prostate cancer and their partners. The public or short title is: Navigate. Ethics approval has been granted by the Peter MacCallum Cancer Centre Human Research Ethics Committee, with site-specific approval from each recruitment site. Approval has also been granted on behalf of the Commonwealth Department of Human Services to obtain Medicare data from Navigate participants who provide consent to do so. Informed written consent is being obtained from all participants. Protocol changes will be communicated to all participating sites. 


\section{Consent for publication}

Not applicable as no identifying information of participants will be published.

\section{Competing interests}

The authors declare that they have no competing interests.

\section{Author details}

'Department of Psychology, Swinburne University of Technology, Melbourne, Victoria, Australia. ${ }^{2}$ Behavioural Science Unit, Peter MacCallum Cancer Centre, Melbourne, Victoria, Australia. ${ }^{3}$ Sir Peter MacCallum Department of Oncology, The University of Melbourne, Parkville, Victoria, Australia. ${ }^{4}$ Swinburne University of Technology, John Street, Hawthorn, Australia. ${ }^{5}$ Department of Nursing, The University of Melbourne, Parkville, Victoria, Australia. ${ }^{6}$ Department of Urology, Cabrini Institute, Cabrini Health, Malvern, Australia. ${ }^{7}$ Department of Surgery, Monash University, Melbourne, Victoria, Australia. ${ }^{8}$ Faculty of Health, University of Technology Sydney, Sydney, Australia. ${ }^{9}$ Health and Wellness Institute, Edith Cowan University, Perth, Australia. ${ }^{10}$ Institute for Resilient Regions, University of Southern Queensland, Springfield, Australia. " Menzies Health Institute Queensland, Griffith University, Southport, Australia. ${ }^{12}$ Population Health Department, Health Economics, QIMR Berghofer Medical Research Institute, Brisbane, Queensland, Australia. ${ }^{13}$ School of Nursing, Queensland University of Technology (QUT), Brisbane, Queensland, Australia. ${ }^{14}$ School of Public Health, University of Queensland, Brisbane, Queensland, Australia. ${ }^{15}$ School of Medicine, University of Queensland, Brisbane, Queensland, Australia. ${ }^{16}$ Department of Urology, Royal Brisbane \& Women's Hospital, Herston, Queensland, Australia.

${ }^{17}$ Division of Cancer Surgery, Peter MacCallum Cancer Centre, Melbourne, Victoria, Australia. ${ }^{18}$ School of Science, RMIT University, Melbourne, Victoria, Australia. ${ }^{19}$ Department of Psychology, The University of Melbourne, Parkville, Victoria, Australia. ${ }^{20}$ Faculty of Health, Deakin University, Bundoora, Victoria, Australia. ${ }^{21}$ Department of Health Science and Biostatistics, Swinburne University of Technology, Melbourne, Victoria, Australia. ${ }^{22}$ School of Psychology, Faculty of Science, Centre for Medical Psychology and Evidence-based Decision-making (CeMPED), University of Sydney, Sydney, New South Wales, Australia.

Received: 24 July 2020 Accepted: 18 December 2020

Published online: 11 January 2021

\section{References}

1. Ferlay J, Soerjomataram I, Dikshit R, et al. Cancer incidence and mortality worldwide: sources, methods and major patterns in GLOBOCAN 2012. Int Cancer. 2015;136(5):E359-E86.

2. Zargar H, van den Bergh R, Moon D, Lawrentschuk N, Costello A, Murphy D. The impact of the United States Preventive Services Task Force (USPTSTF) recommendations against prostate-specific antigen (PSA) testing on PSA testing in Australia. BJU Int. 2017;119:10-115.

3. Wang LL, Begashaw K, Evans M, et al. Patterns of care and outcomes for men diagnosed with prostate cancer in Victoria: an update. ANZ J Surg. 2018:88(10):1037-42

4. Savage CJ, Lilja H, Cronin AM, Ulmert D, Vickers AJ. Empirical estimates of the lead time distribution for prostate cancer based on two independent representative cohorts of men not subject to PSA-screening. Cancer Epidemiol Biomark Prev. 2010;19(5):1201-7.

5. Wang LL, Wallis CJD, Sathianathen N, et al. 'ProtecTion' from overtreatment: does a randomized trial finally answer the key quesiton in localized prostate cancer? BJU Int. 2017;119:513-4.

6. Hamdy FC, Donovan JL, Lane JA, et al. 10-year outcomes after monitoring, surgery, or radiotherapy for localized prostate cancer. N Engl J Med. 2016; 375(15):1415-24.

7. Venderbos LDF, Aluwini S, Roobol MJ, et al. Long-term follow-up after active surveillance or curative treatment: quality-of-life outcomes of men with low-risk prostate cancer. Qual Life Res. 2017;26(6):1635-45.

8. Zelefsky MJ, Poon BY, Eastham J, Vickers A, Pei X, Scardino PT. Longitudinal assessment of quality of life after surgery, conformal brachytherapy, and intensity-modulated radiation therapy for prostate cancer. Radiother Oncol. 2016;118(1):85-91.

9. Soloway MS, Soloway CT, Eldefrawy A, Acosta K, Kava B, Manoharan M. Careful selection and close monitoring of low-risk prostate cancer patients on active surveillance minimizes the need for treatment. Eur Urol. 2010; 58(6):831-5.

10. Briganti A, Fossati N, Catto JWF, et al. Active surveillance for low-risk prostate cancer: the European Association of Urology Position in 2018. Eur Urol. 2018;74(3):357-68.

11. Weerakoon M, Papa N, Lawrentschuk $N$, et al. The use of active surveillance in an Australian cohort of men: a pattern of care analysis from the Victorian Prostate Cancer Registry. BJU Int. 2015;15:S50-S6.

12. Heidenreich A, Bastian PJ, Bellmunt J, et al. EAU guidelines on prostate cancer. Part 1: screening, diagnosis, and local treatment with curative intent - update 2013. Eur Urol. 2014;65(1):124-37.

13. Evans SM, Millar JL, Davis ID, et al. Patterns of care for men diagnosed with prostate cancer in Victoria from 2008 to 2011. Med J Aust. 2013;198(10): 540-5.

14. Murphy DG, Loeb S. Prostate cancer: growth of AS in the USA signals reduction in overtreatment. Nat Rev Urol. 2015;12(11):604-5.

15. McFall SL, Mullen PD, Byrd TL, et al. Treatment decisions for localized prostate cancer: a concept mapping approach. Health Expect. 2015;18(6): 2079-90.

16. Cooperberg MR, Carroll PR, Klotz L. Active surveillance for prostate cancer: progress and promise. J Clin Oncol. 2011;29(27):3669-76.

17. Ganz PA, Barry JM, Burke W, et al. National Institutes of Health State-of-theScience Conference: role of active surveillance in the management of men with localized prostate cancer. Ann Intern Med. 2012;156(8):591-5.

18. Gordon LG, Tuffaha HW, James R, et al. Estimating the healthcare costs of treating prostate cancer in Australia: a Markov modelling anlaysis. Urol Oncol. 2018;36(3):91 e7 - e15.

19. Miller KD, Siegel RL, Lin CC, et al. Cancer treatment and survivorship statistics, 2016. CA Cancer J Clin. 2016;66(4):271-89.

20. Lu-Yao GL, Albertsen PC, Moore DF, Lin Y, DiPaola RS, Yao S-L. Fifteen-year outcomes following conservative management among men aged 65 years or older with localized prostate cancer. Eur Urol. 2015;68(5):805-11.

21. Evans MA, Millar $J$, Earnest $A$, et al. Active surveillance of men with low risk prostate cancer: evidence from the Prostate Cancer Outcomes RegistryVictoria. Med J Aust. 2018;208(10):439-43.

22. Jeldres C, Cullen J, Hurwitz LM, et al. Prospective quality-of-life outcomes for low-risk prostate cancer: active surveillance versus radical prostatectomy. Cancer. 2015;121(14):2465-73.

23. Steginga SK, Occhipinti S, Gardiner RF, Yaxley J, Heathcote P. Prospective study of men's psychological and decision-related adjustment after treatment for localized prostate cancer. Urology. 2004;63(4):751-6.

24. O'Callaghan C, Dryden T, Hyatt A, et al. 'What is this active surveillance thing?' Men's and partners' reactions to treatment decision making for prostate cancer when active surveillance is the recommended treatment option. Psycho-oncology. 2014;23(12):1391-8.

25. Hyatt A, O'Callaghan C, Dryden T, et al. Australian men with low risk prostate cancer and their partners' experience of treatment decision-making and active surveillance. BJU Int. 2013;112:54-65.

26. Violette PD, Agoritsas T, Alexander P, et al. Decision aids for localized prostate cancer treatment choice: systematic review and meta-analysis. CA Cancer J Clin. 2015;65:239-51.

27. Hoffman RM. Improving the communication of benefits and harms of treatment strategies: decision aids for localized prostate cancer treatment decisions. JNCI Monographs. 2012;2012(45):197-201.

28. Zeliadt SB, Ramsey SD, Penson DF, et al. Why do men choose one treatment over another? Cancer. 2006;106(9):1865-74.

29. Couper J, Bloch S, Love A, Duchesne G, MacVean M, Kissane D. Coping patterns and psychosocial distress in female partners of prostate cancer patients. Psychosomatics. 2009:50(4):375-82.

30. Elwyn G, Stiel M, Durand MA, Boivin J. The design of patient decision support interventions: addressing the theory-practice gap. J Eval Clin Pract. 2011;17(4):565-74

31. Stacey $D$, Légaré $F$, Lewis $K$, et al. Decision aids for people facing health treatment or screening decisions. Cochrane Database Syst Rev. 2017;4 CD001431.

32. Elwyn $\mathrm{G}$, Laitner $\mathrm{S}$, Coulter $\mathrm{A}$, Walker $\mathrm{E}$, Watson $\mathrm{P}$, Thomson $\mathrm{R}$. Implementing shared decision making in the NHS. BMJ. 2010;34:341.

33. Feldman-Stewart $D$, Tong $C$, Siemens $R$, et al. The impact of explicit values clarification exercises in a patient decision aid emerges after the decision is actually made: evidence from a randomized controlled trial. Med Decis Mak. 2012;32(4):616-26 
34. van Tol-Geerdink JJ, Willem Leer J, Weijerman PC, et al. Choice between prostatectomy and radiotherapy when men are eligible for both: a randomized controlled trial of usual care vs decision aid. BJU Int. 2013; 111(4):564-73.

35. Berry DL, Halpenny B, Hong F, et al. The Personal Patient Profile-Prostate decision support for men with localized prostate cancer: a multi-center randomized trial. In: Urologic Oncology: Seminars and Original Investigations: Elsevier; 2013.

36. Sidana A, Hernandez DJ, Feng Z, et al. Treatment decision-making for localized prostate cancer: what younger men choose and why. Prostate. 2012;72(1):58-64.

37. Australian Institute of Health and Welfare. Australia's health 2018. Canberra: Austalia AlHW; 2018.

38. Bennett C, Graham ID, Kristjansson E, Kearing SA, Clay KF, O'Connor AM. Validation of a preparation for decision making scale. Patient Educ Couns. 2010;78(1):130-3.

39. O'Connor AM. Validation of a decisional conflict scale. Med Decis Mak. 1995; 15(1):25-30.

40. Holmes-Rovner M, Kroll J, Schmitt N, et al. Patient satisfaction with health care decisions the satisfaction with decision scale. Med Decis Mak. 1996; 16(1):58-64

41. Arden-Close E, Moss-Morris R, Dennison L, Bayne L, Gidron Y. The Couples" Illness Communication Scale (CICS): development and evaluation of a brief measure assessing illness-related couple communication. Br J Health Psychol. 2010;15(3):543-59.

42. Garcia SF, Cella D, Clauser SB, et al. Standardizing patient-reported outcomes assessment in cancer clinical trials: a patient-reported outcomes measurement information system initiative. J Clin Oncol. 2007;25(32):510612.

43. Szymanski KM, Wei JT, Dunn RL, Sanda MG. Development and validation of an abbreviated version of the expanded prostate cancer index composite instrument for measuring health-related quality of life among prostate cancer survivors. Urology. 2010;76(5):1245-50.

44. Hurst N, Kind P, Ruta D, Hunter M, Stubbings A. Measuring health-related quality of life in rheumatoid arthritis: validity, responsiveness and reliability of EuroQol (EQ-5D). Rheumatology. 1997;36(5):551-9.

45. Pickard AS, Wilke CT, Lin H-W, Lloyd A. Health utilities using the EQ-5D in studies of cancer. PharmacoEconomics. 2007;25(5):365-84.

46. Gordon LG, Walker SM, Mervin CM, et al. Financial toxicity: a potential sideeffect of prostate cancer treatment among Australian men. Euro J Cancer Care. 2017;26:e12392

47. de Souza JA, Yap BJ, Hlubocky FJ, et al. The development of a financial toxicity patient-reported outcome in cancer: the COST measure. Cancer. 2014;120(20):3245-53.

48. Weinstein MC, O'Brien B, Hornberger J, et al. Principles of good practice for decision analytic modeling in health-care evaluation: report of the ISPOR task force on good research practices-modeling studies. Value Health. 2003;6(1):9-17.

49. Husereau D, Drummond M, Petrou S, et al. Consolidated Health Economic Evaluation Reporting Standards (CHEERS)--explanation and elaboration: a report of the ISPOR Health Economic Evaluation Publication Guidelines Good Reporting Practices Task Force. Value Health. 2013;16(2):231-50.

50. Torre LA, Bray F, Siegel RL, Ferlay J, Lortet-Tieulent J, Jemal A. Global cancer statistics, 2012. CA Cancer J Clin. 2015;65(2):87-108.

\section{Publisher's Note}

Springer Nature remains neutral with regard to jurisdictional claims in published maps and institutional affiliations.

Ready to submit your research? Choose BMC and benefit from:

- fast, convenient online submission

- thorough peer review by experienced researchers in your field

- rapid publication on acceptance

- support for research data, including large and complex data types

- gold Open Access which fosters wider collaboration and increased citations

- maximum visibility for your research: over $100 \mathrm{M}$ website views per year

At BMC, research is always in progress.

Learn more biomedcentral.com/submissions 